\title{
Copula joint function and its application in probability seismic hazard analysis*
}

\author{
LI Yan-heng" (李彦恒) SHI Bao-ping (史保平) ZHANG Jian (张健) \\ College of Earth Science, Graduate University of the Chinese Academy of Sciences, Beijing 100049, China
}

\begin{abstract}
A mature mathematical technique called copula joint function is introduced in this paper, which is commonly used in the financial risk analysis to estimate uncertainty. The joint function is generalized to the $n$-dimensional Frank's copula. In addition, we adopt two attenuation models proposed by YU and Boore et al, respectively, and construct a two-dimensional copula joint probabilistic function as an example to illustrate the uncertainty treatment at low probability. The results show that copula joint function gives us a better prediction of peak ground motion than that resultant from the simple linear weight technique which is commonly used in the traditional logic-tree treatment of model uncertainties. In light of widespread application in the risk analysis from financial investment to insurance assessment, we believe that the copula-based technique will have a potential application in the seismic hazard analysis.
\end{abstract}

Key words: uncertainty; copula function; seismic hazard analysis; attenuation model; lognormal distribution CLC number: P315.9 Document code: A

\section{Introduction}

The probabilistic seismic hazard analysis (PSHA) is to make a quantitative estimate of the likelihood of exceeding a given threshold of earthquake-caused ground motions in a specific region during a given time period and thus provide scientific base for decision-making of earthquake-related engineering design and financial investment. PSHA $^{\mathbb{}(\odot)}(\mathrm{HU}, 2006)$ involves identifications of potential seismic sources region, occurrence rates for all earthquakes in a region, characterizations of attenuation relationships and site effects, etc. Each of the aspects is characterized by deep uncertainty (YAN, 2005), for not only there is uncertainty resulted from approximation of the theory used in the analysis, for example, the occurrence and distribution of real earthquakes may not follow Gutenberg-Richter relation exactly in somewhere; but also man-made subjective uncertainty. The latter is called model uncertainty which will be discussed in this paper. As we know, in order to build a seismic hazard model, many physical parameters have to be taken into account. In this case, the corresponding hypotheses introduced by different experts usually lead to diverse probability distributions in the hazard models. In the implemental level, each

\footnotetext{
* Received 2007-05-21; accepted in revised form 2008-04-02.

Foundation item: Project of Institute of Crustal Dynamics, China Earthquake Administration (ZDJ2007-1), One Hundred Individual Program of Chinese Academy of Sciences (99M2009M02) and National Natural Science Foundation of China (40574022).

* Author for correspondence: liyanh06b@mails.gucas.ac.cn

${ }^{(1)}$ Budnitz R J, Apostolakis G, Boore D M, et al. 1997. Recommendations for probabilistic seismic hazard analysis: Guidance on uncertainty and use of experts [R]. Report prepared for the U.S. Nuclear Regulatory Commission, the U.S. Department of Energy, and the Electric Power Research Institute by the Senior Seismic Hazard Analysis Committee (SSHAC), Report No. NUREG/CR-6372.

(2) Working Group on California Earthquake Probabilities (WGCEP). 2003. Earthquake probabilities in the San Francisco Bay Region: 2002-2031 [R]. Working Group on California Earthquake Probabilities, Tech. Rep. Open-File Report 03 214, USGS.
} 
model could only catch partial real physical information related to the earthquake ground motion. On the other hand, because each model is not independent but inter-related, therefore, the stochastic characteristics of ground motion are not contributed only by a certain factor but also several inter-related factors. In fact, each model only captures some aspects of the underlying processes, so it is necessary to suitably make the best of the merits of every model in the modern seismic hazard analysis. Currently, in the practical procedure, there are three methodologies that can comprehensively assess the effects from model uncertainty: the linear combi-

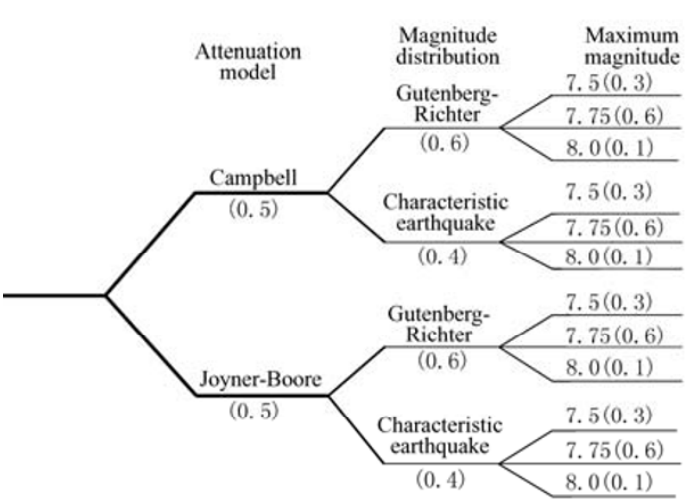

Figure 1 The Logic-Tree commonly applied in the probabilistic seismic hazard analysis The numbers in bracket are weights nation of models, choosing model weights based upon data availability, and arbitrary ordering of models (Kramer, 1996). Logic-tree is widely used in the prevalent PSHA to deal with model uncertainty. The hazard analysis estimated by logic tree with linear weights is shown in Figure 1. It is true that linear average is the easiest way in the hazard estimation. However, the logic tree technique with some subject factors neglects the inter-dependence among different models so as to come to an overrated or underrated conclusion. Therefore, the uncertainties may be amplified in sometime. On the other hand, the weight function of model is strongly dependent on the original data and personal options so that some systemic errors could exist in the final probability distribution. It seems that the resultant linear weight used in the logic-tree technique dose not reduce the model uncertainties effectively.

Copula function technique presented in this paper has not been applied in financial risk assessment until 1999 (Clemen and Winkler, 1999) since it was proposed by Sklar (1959). Before 1999 , the main approach in the financial risk analysis is to assess the risks of the individual financial assets separately, and then combine all of them with some weights. By using copula theory, a number of marginal distributions and a copula function connecting them can be written as a joint distribution closer to the actual raw data so as to establish a more effective risk management model. In addition, the copula function can divide the risks into two parts: the risk from each individual financial asset and another from the investment portfolio based on these assets. It does not only make modeling considerably simplified, but also contribute to analyzing and understanding many intricate financial problems. In recent years, since researches on copula functions and its applications in financial risk analysis are wide and rapid (Bouye et al, 2000; Rockinger and Jondeau, 2002; Embrechts et al, 2002; ZHANG, 2002), we believe that this method can provide us a new treatment for seismic risk analysis analogous to financial scope (Morgan and Carlson, 2006). This joint probability distribution can properly take into account the correlation between the different models, therefore attain a more reasonable and accurate estimation to potential earthquake hazards in a special region.

\section{Introduction of copula function}

1) Definition of copula function

A $n$-dimensional copula is a multivariate distribution function $C$, with uniform distributed 
margins in $[0,1]$ and satisfies the following three conditions:

(1) $C:[0,1]^{n} \rightarrow[0,1]$;

(2) $C$ is grounded and $n$-increasing;

(3) $C$ has margins $C_{i}$ which satisfy $C_{i}(u)=C(1, \cdots, 1, u, 1, \cdots 1)=u_{i}, u_{i} \in[0,1]$.

It is obvious from the above definition that if $F_{1}, \cdots, F_{n}$ are univariate distribution functions, $C\left[F_{1}\left(x_{1}\right), \cdots, F_{n}\left(x_{n}\right)\right]$ is a multivariate distribution function with margins $F_{1}, \cdots, F_{n}$. Copula functions are a useful tool to construct and simulate multivariate distributions.

According to Sklar's theorem (1959) and Probability-Integral Transforms Law, a $n$-dimension distribution can be divided into two parts: uniform marginal distribution and dependent structure, the joint distribution density $f\left(x_{1}, \cdots, x_{n}\right)$ can be deduced as:

$$
f\left(x_{1}, \cdots, x_{n}\right)=\frac{\partial C\left[F_{1}\left(x_{1}\right), \cdots, F_{n}\left(x_{n}\right)\right]}{\partial x_{1} \cdots \partial x_{n}}=c(\widetilde{u}) \prod_{i} f_{i}\left(x_{i}\right)
$$

where $f\left(x_{1}, \cdots, x_{n}\right)$ is the density corresponding to $F(\cdot), \widetilde{u}=\left(u_{1}, \cdots, u_{n}\right), u_{i}=F_{i}\left(x_{i}\right)(i=1, \cdots, n)$, and $c(\widetilde{u})$ is called the copula density. Equation (1) states that, under appropriate conditions, the joint density $f\left(x_{1}, \cdots, x_{n}\right)$ can be written as a product of the marginal densities and the copula density. The former $c(\widetilde{u})$ is copula density, that is, an associated structure with normal random variables $x_{1}, x_{2}, \cdots, x_{n}$, which determines co-movement among these variables. The latter $\prod f_{i}\left(x_{i}\right)(i=1,2, \cdots$, $n)$ is the product of several marginal probability density functions. That is to say, after each marginal distribution $F_{i}\left(x_{i}\right)$ of random variables $x_{i}$ is given and some proper parameters are assessed to accord with every univariate marginal, we can choose an appropriate correction factor, that is, copula function, which is necessary for a joint probability distribution.

2) Properties of copula $C$

(1) $C\left(u_{1}, \cdots, u_{n}\right)=0$, if any $u_{j}=0,1 \leq j \leq n$;

(2) $C\left(1,1, \cdots, u_{j}, \cdots, 1\right)=u_{j}, 1 \leq j \leq n$;

(3) $C\left(u_{1}, \cdots, u_{n}\right)=u_{1} \times \cdots \times u_{n}$ if all $u$ are independent.

3) Concordance

Dependence relation is always one of the most widely studied subjects in statistics. In many of these properties of dependence discussed scale-invariant means that they remain unchanged under strictly increasing transformations of random variables. Copula function just has this property called concordance that under almost surely strictly increasing transformations the corresponding copulas are invariant. Then the measure of dependence reduced from the copulas is unchanged. It implies that the measures from copulas, compared with common measures, i.e., linear correlation coefficient (Pearson's product-moment coefficient), could be applied more widely and practically.

In a general way, Kendall's $\tau$ (Kendall, 1938) and Spearman's $\rho$ (Kruskal, 1958) are labeled as the measurements of concordance besides Gini's coefficient (Rényi, 1959), etc. Especially, the definition of Kendall's $\tau$ is:

$$
\tau_{n}=\frac{1}{2^{n}-1}\left[2^{n} \int_{[0,1]^{n}} C_{n}\left(u_{1}, \cdots, u_{n}\right) \mathrm{d} C_{n}\left(u_{1}, \cdots, u_{n}\right)-1\right]
$$

4) General copulas

(1) Multivariate normal copula 
Given $\Sigma$ is a real positive symmetric matrix and $\operatorname{diag}(\Sigma)=(1,1, \cdots, 1) . \Phi_{\Sigma}$ is a standard multivariate normal distribution with a correlation matrix $\Sigma$. $\Phi$ is a standard cumulative probability distribution of Gaussian variable $x_{i}$. As the definition above, a multivariate normal copula distribution function is written as:

$$
C\left(u_{1}, u_{2}, \cdots, u_{n} ; \Sigma\right)=\Phi_{\Sigma}\left[\Phi^{-1}\left(u_{1}\right), \Phi^{-1}\left(u_{2}\right), \cdots, \Phi^{-1}\left(u_{n}\right)\right]
$$

Let $\boldsymbol{\Phi}\left(x_{i}\right)$ is equal to marginal distribution $u_{i}\left(y_{i}\right)$ of random variable $y_{i}$, then $x_{i}=\Phi^{-1}\left(u_{i}\right)$, equation (3) can be rewritten and the density coefficient function of multivariate normal copula would be

$$
c\left(u_{1}, u_{2}, \cdots, u_{n}\right)=\frac{1}{|\boldsymbol{\Sigma}|^{1 / 2}} \exp \left[-\frac{1}{2} \boldsymbol{\zeta}^{\prime}\left(\boldsymbol{\Sigma}^{-1}-\boldsymbol{I}\right) \boldsymbol{\zeta}\right]
$$

where $\zeta=\left[\boldsymbol{\Phi}^{-1}\left[u_{1}\left(y_{1}\right)\right], \boldsymbol{\Phi}^{-1}\left[u_{2}\left(y_{2}\right)\right], \cdots, \boldsymbol{\Phi}^{-1}\left[u_{n}\left(y_{n}\right)\right]\right]^{\prime}$.

(2) Archimedean copula (Nelsen, 1998)

A $n$-dimension copula $C$ is Archimedean copula, if only if there exists a continuous and strictly decreasing function $\psi \cdot[0,+\infty] \rightarrow[0,1]$ with $\psi(0)=1$, and a function $\phi$ defined by

$$
\psi(t)= \begin{cases}\phi^{-1}(t) & t \in[0, \phi(0)] \\ 0 & t \in[\phi(0),+\infty]\end{cases}
$$

For all $\left(u_{1}, \cdots, u_{n}\right) \in[0,1]^{n}, C\left(u_{1}, \cdots, u_{n}\right)=\psi\left[\phi\left(u_{1}\right)+\cdots+\phi\left(u_{n}\right)\right] . \phi$ is called generating function of copula $C$. Furthermore, if $C$ strictly increases in $[0,1]^{n}, \psi$ and $\phi^{-1}$ vary in the same way in all $[0$, $+\infty]$. The following are some generating functions of common Archimedean copulas.

Frank's copula (Frank, 1979)

$$
\phi(u)=\log _{\alpha}\left[1-(1-\alpha) \mathrm{e}^{-u}\right] \quad \alpha \in[0,1]
$$

Gumbel's copula (Gumbel, 1960)

$$
\phi(u)=[-\ln (u)]^{\alpha} \quad \alpha>1
$$

Cook and Johnson's copula (Clayton, 1978)

$$
\phi(u)=u^{-\alpha}-1 \quad \alpha>0
$$

Before giving a concrete example, we need introduce the Frank's copula and some of its properties in detail.

$$
C_{\alpha}\left(u_{1}, u_{2}, \cdots, u_{n}\right)=\log _{\alpha}\left[1+\frac{\prod_{i=1}^{n}\left(\alpha^{u_{i}}-1\right)}{(\alpha-1)^{n-1}}\right] \quad \alpha \in[0,1]
$$

From equation (8), we deduce $n$-dimension copula joint probability density function as

$$
c_{n, \alpha}=\left(\frac{\ln \alpha}{\alpha-1}\right)^{n-1} \frac{\prod_{i=1}^{n} \alpha_{i}^{u}}{\left[1+\frac{\prod_{i=1}^{n}\left(\alpha_{i}^{u}-1\right)}{(\alpha-1)^{n-1}}\right]^{n}}\left\{\sum_{j=1}^{n} q_{j-1}(n)\left[1+\frac{\prod_{i=1}^{n}\left(\alpha_{i}^{u}-1\right)}{(\alpha-1)^{n-1}}\right]^{n-j}\right\} \prod_{k=1}^{n}\left[\frac{\alpha_{k}^{u}-1}{(\alpha-1)^{n}}\right]^{j-1}
$$


where $q_{0}(n)=1, q_{i}(n+1)=(i+1) q_{i}(n)-i q_{\mathrm{i}-1}(n), n \geq 1,0 \leq i \leq n-1, q_{i}(i)=0$. The copula family of Frank can capture the full range of positive dependence. The dependence level among several probability distributions is indexed by a single parameter, Kendall's $\tau$. When $n=2$,

$$
\tau_{2}=\frac{2}{9(\ln \alpha)^{2}}\left[\pi^{2}+6 \ln \alpha-6 \ln (1-\alpha) \ln \alpha-6 \text { polylog }[2, \alpha]\right]+\frac{1}{3}
$$

where polylog $[2, \alpha]$ is Riemann function. If $\tau_{2}=0$, all the models are independent; while if $\tau_{2}=1$, they are perfectly positive dependence. Frank's copula treats all its marginal distributions symmetrically.

\section{Applications}

In this section we give a simple example to demonstrate how Frank's copula is applied to the probability seismic hazard analysis. Due to lack of adequate and prefect strong-ground-motion data measured by the modern instrument in some great earthquakes, empirical ground motion attenuation models are nowadays rested on the criterions for earthquake-resistant engineering, architecture designing, the seismic hazard mapping, and other relative fields. These models with several parameters of ground motion (peak ground acceleration, velocity, displacement, response spectrum, etc.) varying as earthquake magnitude and source-site distance are founded on the regression analysis elicited from few sets of data of strong earthquakes. The ground motion attenuation curves offer geometric medians and degrees of uncertainty, standard deviate $\sigma$ reflecting the bias between theoretical geometric median and actual observed values.

In a general way, considering earthquake magnitude $M$ and source-site distance $R$, we can get the probability density function of exceeding each ground motion parameter $y$ as

$$
p(\log y)=\frac{1}{\sigma \sqrt{2 \pi}} \exp \left\{\frac{[\log y-\log Y(M, R)]^{2}}{2 \sigma^{2}}\right\}
$$

$Y(M, R)$ is a ground motion attenuation curve from data analysis, and $\sigma$ is the corresponding standard deviate. Thus the resulting cumulative exceedance probability of $y \geq X$ is

$$
\Phi[y \geq X \mid Y(M, R, \sigma)]=\frac{1}{2} \operatorname{erfc}\left[\frac{\log X-\log Y(M, R)}{\sqrt{2 \sigma}}\right]
$$

where $\operatorname{erfc}(x)$ is a complementary error function. In terms of seismic data from a certain region, each expert might respectively give a different attenuation model. In modern PSHA, the more expert's models are employed, the more reasonable and reliable the result is. As an international rule, it is a few of experts' models that the hazard assessment employs in the crucial infrastructure [e.g., about seven experts' models were used in Bernreuter et al (1989)]. Whereas in this paper, we would only apply two different seismic ground motion attenuation models as a concrete example. Figure 2 illustrates the attenuation curves provided by YU (2002) and Boore et al (1997). Although the two models use the same set of seismic data from California, the results from them have distinct discrepancy. For instance, in view of a given magnitude in the near-fault distance $(\leq 10 \mathrm{~km})$, the variance of peak ground acceleration is as large as $3.0 \mathrm{~m} / \mathrm{s}^{2}$. Besides this, their standard deviations are respectively $4.11 \mathrm{~m} / \mathrm{s}^{2}$ and $5.20 \mathrm{~m} / \mathrm{s}^{2}$.

Based on the Frank's copula, for earthquake with magnitude of $M 8.0$ at a distance of $R=20$ $\mathrm{km}$, two lognormal distributions with the median PGAs of $5.0 \mathrm{~m} / \mathrm{s}^{2}$ and $3.5 \mathrm{~m} / \mathrm{s}^{2}$ respectively from 
the two ground motion attenuation models are combined. Equations (2) and (11) can present a joint probability density function at $\tau=0, \tau=0.3, \tau=0.75$, compared with an equally weighted linear combination. The difference between the two expert-aggregation methods should be illustrated in Figure 3 and Table 1. The equally weighted average takes all the information from the two experts into account indistinctively so that the uncertainty included by the average distribution is enlarged: in Figure 3a the standard deviate $\left(\sigma=5.03 \mathrm{~m} / \mathrm{s}^{2}\right)$ of the average distribution is not smaller than both of the two experts. In addition, it assigns too

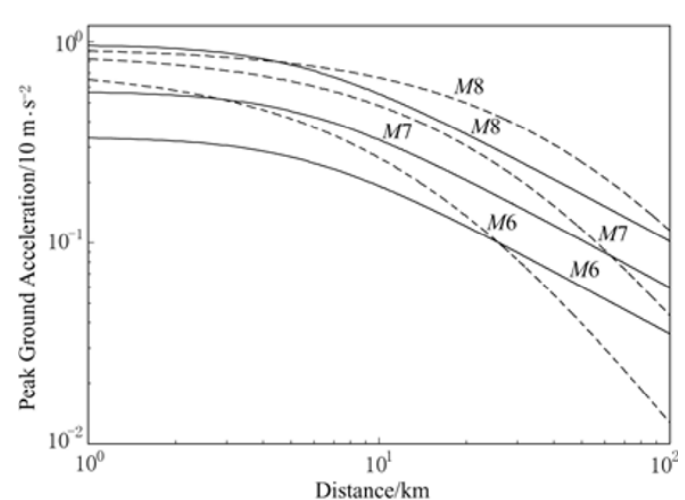

Figure 2 A comparison of attenuation models derived both from YU (dashed lines) and Boore (solid lines) little a role in the distribution with the smaller deviate. An aggregation from several experts' advices, intuitively (Shafer, 1976), should be more informative than knowledge from every expert. However, in term of general weighted methods, $P=\sum_{i=1}^{n} w_{i} P_{i}, \sum_{i=1}^{n} w_{i}=1, w_{i} \in[0,1]$, for any $w_{i}$

$$
\min _{1 \leq i \leq n} P_{i} \leq \sum_{i=1}^{n} w_{i} P_{i} \leq \max _{1 \leq i \leq n} P_{i}
$$

Equation (13) is not consistent with our ordinary intuitions, because this linear combination, in reality, considers two sets of information obtained from the two distributions as a whole without accounting for the overlapping part in them. Whereas both of the attenuation models in our paper actually are based on the modern seismologic instrumental data in California, U.S.A. If the two distributions are independent, Kendall's $\tau$ equals zero and our copula density function $c\left(u_{1}, u_{2}\right)$ reduces to 1 as shown in Figure 3b. In this case the new distribution enhances certainty in the uncertain region of the two experts' opinions, which underestimates the true hazard due to the decrease of standard deviate $\left(\sigma=2.33 \mathrm{~m} / \mathrm{s}^{2}\right.$ ). In the cases of $\tau=0.30$ and $\tau=0.75$ shown by Figures $3 \mathrm{c}$ and $3 \mathrm{~d}$, these copula-based joint distributions might be measurable indicator of an virtue that two experts could bring us more information than an expert. The copula-aggregation distributions almost spread in a certain scale $\left[1.0 \mathrm{~m} / \mathrm{s}^{2}, 12.0 \mathrm{~m} / \mathrm{s}^{2}\right]$, especially when $\tau=0.3$, the standard deviate $\sigma_{c}$ is only $3.79 \mathrm{~m} / \mathrm{s}^{2}$. As a matter of fact, the distributions produced by two experts are dependent insomuch as the two attenuation models are traced back to the earthquake data from the same area,

Table 1 Means, standard deviations, and the corresponding upper bounds of error bar for the four different distributions

\begin{tabular}{ccccc}
\hline Corresponding statistic value & YU & Boore et al & Average with equal weights & Copula aggregation with $\tau=0.3$ \\
\hline Mean $\bar{m}$ & 5.0 & 3.5 & 4.2 & 4.5 \\
Standard deviation $\sigma$ & 4.1 & 5.2 & 5.0 & 3.8 \\
$\bar{m}+\sigma$ & 7.5 & 5.9 & 6.9 & 6.5 \\
$\bar{m}+2 \sigma$ & 11.4 & 9.8 & 11.4 & 9.5 \\
$\bar{m}+3 \sigma$ & 17.2 & 16.7 & 18.8 & 13.9 \\
\hline
\end{tabular}



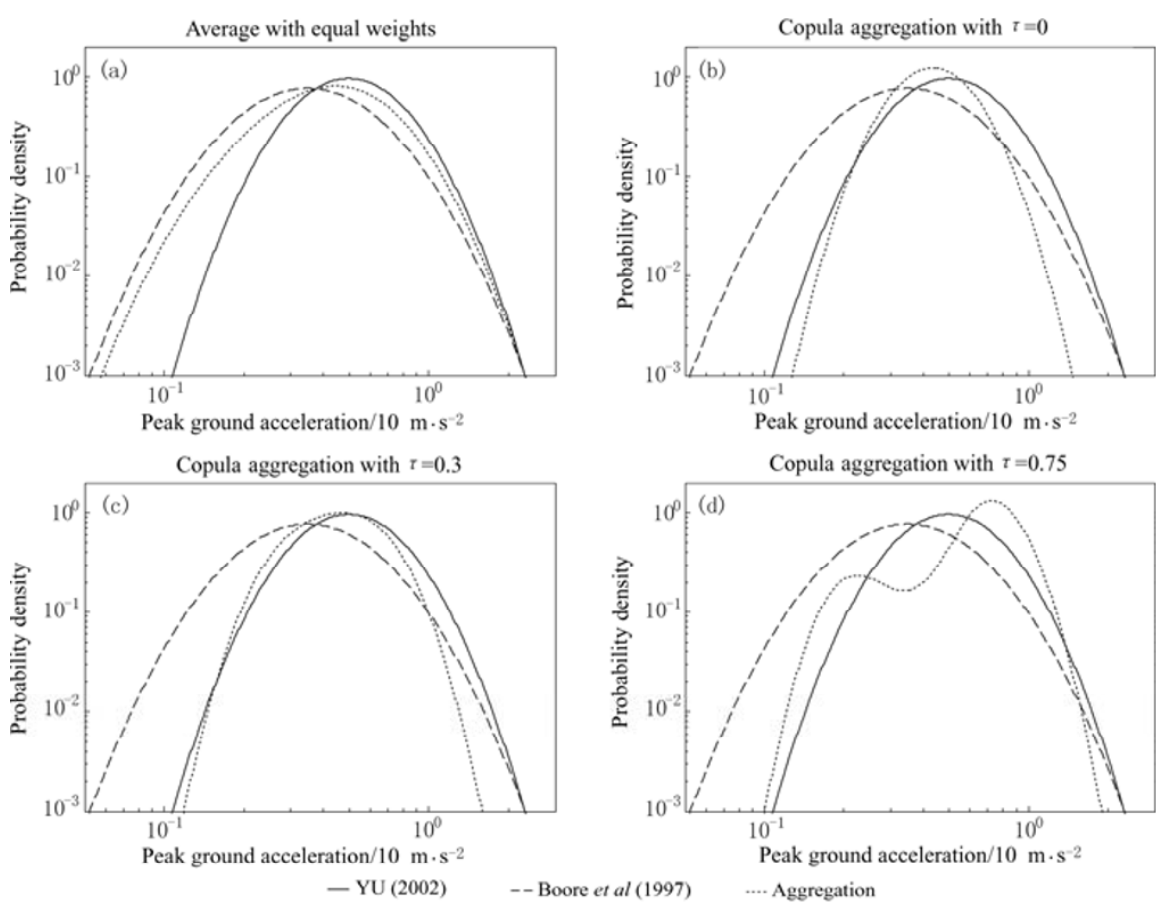

Figure 3 Related peak ground accelerations (mean) and corresponding standard deviations derived from YU's and Boore's attenuation models for the earthquake with magnitude of M8.0 at a distance of $R=20 \mathrm{~km}$

that is, an information source for the two models with similar statistic traits. According to Dempster's law, copula density function draws out the consistent part of all the experts' opinions and filtrate the incompatible in them.

Furthermore, assuming the earthquake scenarios which have its own magnitude M8.0, source-site distance $R=20 \mathrm{~km}$, and annual of rate 0.01 , we get the annual rate at which each logPGA is exceeded due to the scenario:

$$
\lambda(y)=0.01 \Phi[y \geq X \mid Y(M, R, \sigma)]
$$

Figure 4 and Table 2 present in the different recurrence periods peak ground accelerations obtainned from YU's, Boore's, the equal weighted combination between them and the copula-based distribution with $\tau=0.3$. Consequently it is more obvious that in the lower probability of exceedance the copula method is preferable to logic-tree. In the case of $\tau=0.30$, the copula-aggregation distribution is partial to YU's model in respect that the copula emphases the marginal with less variance. Aside from that, the copula joint distribution spreads between the two distributions at the high

Table 2 The predicted peak ground accelerations with $10 \%, 5 \%, 2 \%, 0.5 \%, 0.05 \%$ probabilities with 50 years for the four distributions

\begin{tabular}{ccccc}
\hline Recurrence period/a & YU & Boore et al & Average with equal weights & Copula aggregation with $\tau=0.3$ \\
\hline 500 & 7.1 & 5.4 & 6.4 & 6.2 \\
1000 & 8.5 & 6.8 & 7.8 & 7.3 \\
2500 & 10.3 & 8.7 & 9.7 & 8.5 \\
10000 & 13.1 & 11.7 & 12.5 & 10.2 \\
100000 & 17.9 & 17.4 & 17.8 & 12.9 \\
\hline
\end{tabular}




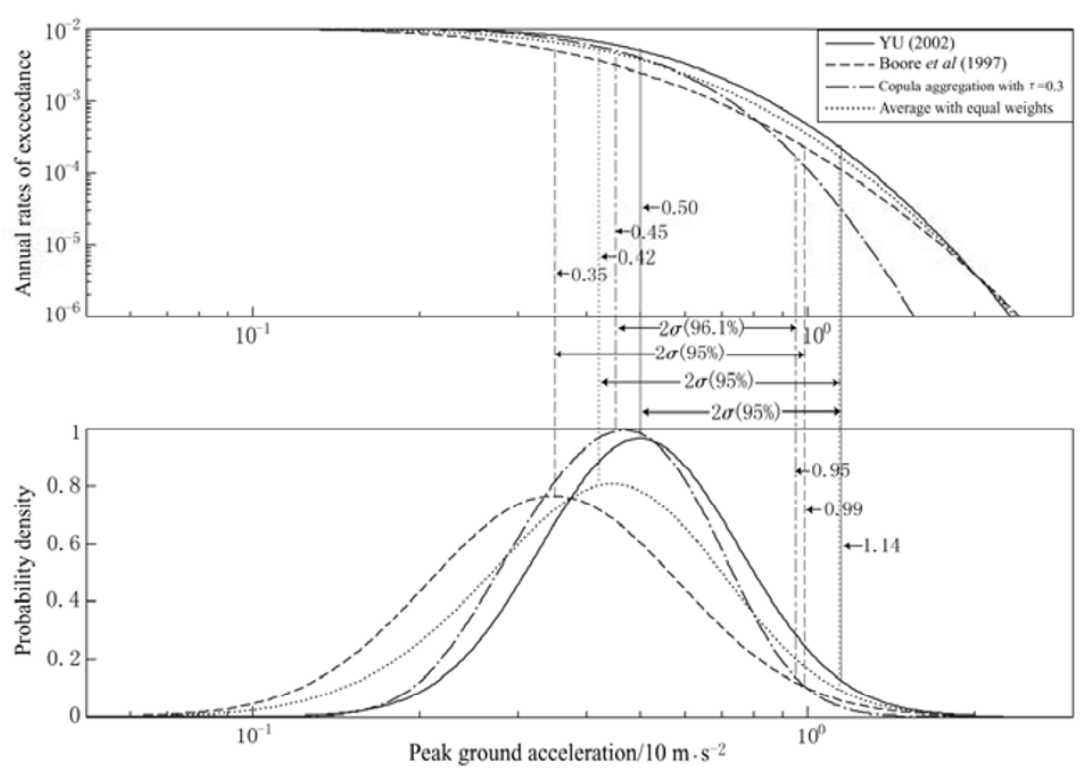

Figure 4 A comparison of different probability density distributions and annual rates of exceedance of PGA for M8.0, and $R=20 \mathrm{~km}$ according to YU 's and Boore's attenuation models A linear combination model with an equally weight and a copula-based aggregation model with $\tau=0.30$ are used for comparison purpose

event probability of exceedance where the copula suitably considers Boore's opinion. In view of the extreme low event probability $(<0.0001)$, the copula joint distribution provides a smaller predicted value of peak ground acceleration (about $20 \%$ smaller than other models) in the quite same confidence level (nearby $96 \%$ ). It is actually $5.0 \mathrm{~m} / \mathrm{s}^{2}$ smaller that the copula is than other distributions at the lower event probability $(<0.00001)$.

In addition, we give another example of magnitude M7.0, source-site distance $50 \mathrm{~km}$ (Figure 5). In the larger distance $(50 \mathrm{~km})$, a little gap between the two experts' predicted values and a low probability where possible great strong ground motion always occurs serve to a little attenuation of the seismologists. At the same time, the near medians from the two aggregation methods are also close to the two experts'. In the last analysis, the copula-based method can also capture the mutual information provided by the two experts in the larger distance. Besides, most of importance is attached into the probability of the predicted median values and peak ground accelerations of the near-fault strong ground motion. Moreover, in order to diminish the discrepancy of median values between the two experts, the copula projects a better aggregation methodology.

\section{Conclusions}

It is a notorious puzzle for human beings how to estimate the seismic hazard in virtue of known mathematical and physical statistics and finite observed data owing to the intricate and elusive uncertainty of the seismic hazard. In this study, after comparisons of average with equal weights and copula aggregation applied in the PSHA, we could safely point out that copula-based method models the dependent experts' opinions more suitable and reliable than the traditional logic-tree method wherever in any event of a low or high probability. We should convict that this paper is merely a brief demonstration in respect that the copula-based method is adapted in mod- 
eling the uncertainty in some multivariable distributions and it will have a potential application in many other risk analysis. In addition, it is not avoidable to refer to how to judge the optimization of copula joint function in the future applications, which require parameter estimations to all raw data, traced by different models and then make a determination by goodness of fit test.
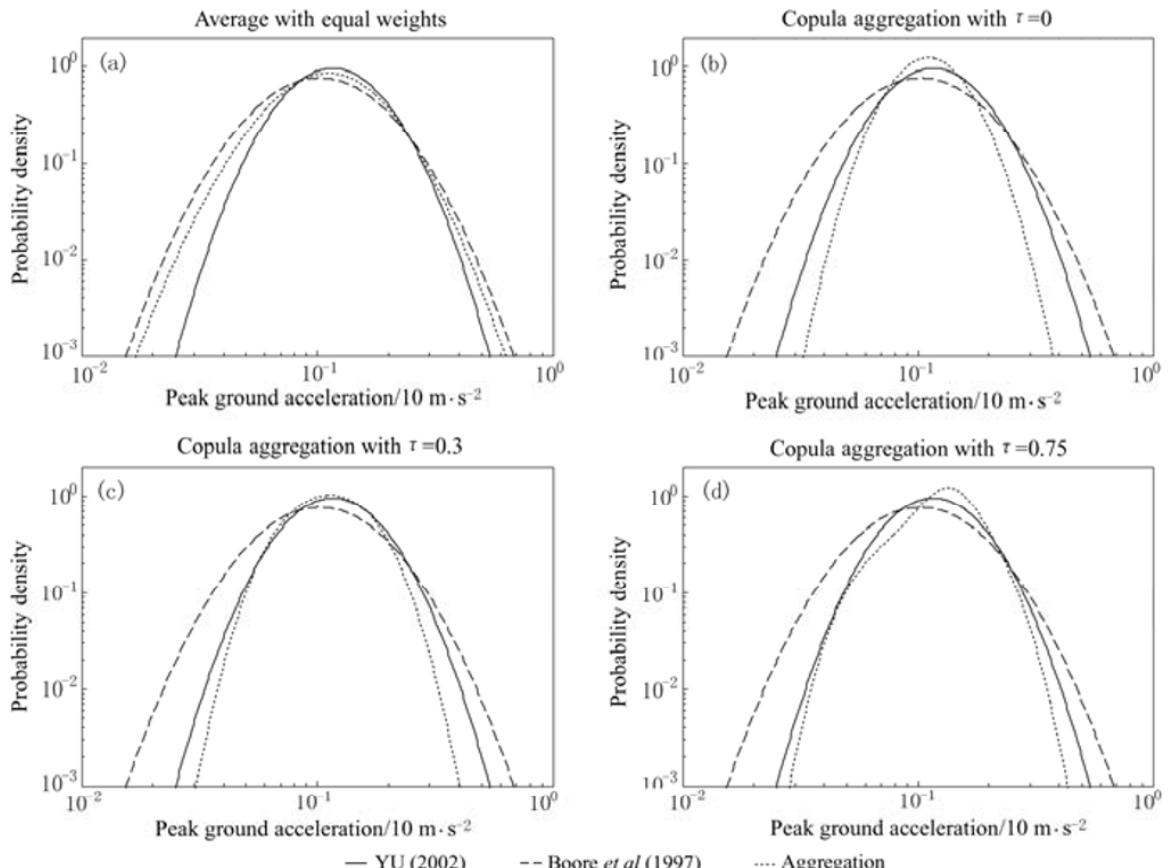

Figure 5 Related peak ground accelerations (mean) and corresponding standard deviations derived from YU's and Boore's attenuation models for the earthquake with magnitude of $M 7.0$ at a distance of $R=50 \mathrm{~km}$

\section{References}

Bernreuter D L, Savy J B, Mensing R W, et al. 1989. Seismic Hazard Characterization of 69 Nuclear Sites East of the Rocky Mountains [R]. Washington D C: U.S. Nuclear Regulatory Commission, Vol.1-8, NUREG/CR 5250, UCID-21517.

Boore D M, Joyner W B, Fumal T E. 1997. Equations for estimating horizontal response spectra and peak acceleration from Western North American earthquakes: A summary of recent work [J]. Seism Res Lett, 68(1): 128-153.

Bouye E, Durrleman V, Nikeghbali A. 2000. Copulas for Finance: A Reading Guide and Some Application [R]. Paris: Groupe de Recherche Operationnelle.

Clayton D G. 1978. A model for association in bivariate life tables and its application in epidemiological studies of familial tendency in chronic disease incidence [J]. Biometrika, 65: 141-151.

Clemen R T and Winkler R L. 1999. Combining probability distributions from experts in risk analysis [J]. Risk Analysis, 19: 187-203.

Embrechts P, McNeal A J, Straumann D. 2002. Correlation and dependence in risk management: Properties and pitfalls [C]//Risk Management: Value at Risk and Beyond. Cambridge: Cambridge University Press: 176-223.

Frank M J. 1979. On the simultaneous associativity of $F(x, y)$ and $x+y-F(x, y)$ [J]. Aeq Math, 19: 194-226.

Gumbel E J. 1960. Distributions des valeurs extrêmes en plusiers dimensions [J]. Pub Inst Stat Univ Paris, 9: 171-173.

HU Yu-xian. 2006. Earthquake Engineering [M]. Beijing: Seismological Press: 354-355 (in Chinese).

Kendall M G. 1938. A new measure of rank correlation [J]. Biometrika, 30: 81-93.

Kramer S L. 1996. Geotechnical Earthquake Engineering [M]. Upper Saddle River N J: Prentice Hall: 137.

Kruskal W H. 1958. Ordinal measures of association [J]. J Amer Statist Assoc, 53: 814-861.

Morgan T P and Carlson J M. 2006. Methodologies for earthquake hazard assessment: Model uncertainty and the WGCEP-2002 forecast [J]. Bull Seism Soc Amer, 96: 1 624-1 633.

Nelsen R B. 1998. An Introduction to Copulas [M]. New York: Springer-Verlag: 37-43.

Rényi A. 1959. On measures of dependence [J]. Acta Math Acad Sci Hungar, 10: 441-451.

Rockinger M and Jondeau E. 2002. Conditional Dependency of Financial Series: An Application of Copulas [R]. Paris: HEC School of 
Management

Shafer G. 1976. A Mathematical Theory of Evidence [M]. Princeton: Princeton University Press: 10-40.

Sklar A. 1959. Fonctions de répartition àn dimensions et leurs marges [J]. Pub Inst Stat Univ Paris, 8: 229-231.

YAN Jia-quan. 2005. Confusion and hope in seismic risk analysis [J]. Recent Developments in World Seismology, 6: 14-24 (in Chinese).

YU Yan-xiang. 2002. Studies on Long-period Seismic Ground-motion Attenuation Relation [D][Ph D Dissertation]. Beijing: Institute of Geophysics, China Seismological Bureau, 110-119 (in Chinese).

ZHANG Yao-ting. 2002. Copula technique in financial risk analysis [J]. Statistical Research, 4: 48-51 (in Chinese). 